\title{
Connection Between Wave Functions in the Dirac and Foldy-Wouthuysen Representations
}

\author{
Alexander J. Silenkd* \\ Institute of Nuclear Problems, Belarusian State University, Minsk 220080, Belarus
}

(Dated: June 20, 2012)

\begin{abstract}
The connection between wave functions in the Dirac and Foldy-Wouthuysen representations is found. When the Foldy-Wouthuysen transformation is exact, upper spinors in two representations differ only by constant factors, and lower spinors in the Foldy-Wouthuysen representation are equal to zero.
\end{abstract}

PACS numbers: 03.65.Pm, 11.10.Ef, 12.20.Ds

Keywords: relativistic quantum mechanics, Foldy-Wouthuysen representation, unitary transformations

*Electronic address: silenko@inp.minsk.by 


\section{INTRODUCTION}

Determination of connection between wave functions in the Dirac and Foldy-Wouthuysen (FW) representations is a very important problem. The Hamiltonian for relativistic particles

in the FW representation contains a square root of operators (see Refs. [1, 2]). Therefore, the Dirac representation is usually more convenient than FW one for finding wave eigenfunctions and eigenvalues of the Hamilton operator. However, the form of operators is much simpler just in the FW representation. For example, in this representation $\boldsymbol{r}$ is the coordinate operator, $\boldsymbol{p}=-i \nabla$ is the momentum operator, $\boldsymbol{l}=\boldsymbol{r} \times \boldsymbol{p}$ is the orbital angular momentum operator, the matrix $\Sigma / 2$ is the spin momentum operator (see Ref. [1]). The polarization operator in the FW representation is the matrix $\Pi[3]$. In the Dirac representation, all corresponding operators are defined by cumbersome expressions [1]. For particles interacting with external fields, these operators also depend on the external field parameters.

The FW representation is very useful to derive semiclassical equations of particle and spin motion [2].

The determination of connection between wave functions in the Dirac and FW representations makes it possible to calculate wave functions only in one of these representations. In the present work, the connection between wave functions is derived when the FW representation is exact.

Throughout the work we use the system of units $\hbar=c=1$ and generally accepted designations of Dirac matrices (see [1] ).

\section{THE EXACT FOLDY-WOUTHUYSEN TRANSFORMATION}

In the general case, the transformation to a new representation described by the wave function $\Psi^{\prime}$ is performed with the unitary operator $U$ :

$$
\Psi^{\prime}=U \Psi
$$

where $\Psi$ is the wave function (bispinor) in the Dirac representation. The Hamilton operator in the new representation takes the form [1, 4]

$$
\mathcal{H}^{\prime}=U \mathcal{H} U^{-1}-i U \frac{\partial U^{-1}}{\partial t}
$$


The Hamiltonian can be split into operators commuting and noncommuting with the operator $\beta$ :

$$
\mathcal{H}=\beta m+\mathcal{E}+\mathcal{O}, \quad \beta \mathcal{E}=\mathcal{E} \beta, \quad \beta \mathcal{O}=-\mathcal{O} \beta
$$

The FW transformation is exact if the external field is stationary and the operators $\mathcal{E}$ and $\mathcal{O}$ commute $[2]$ :

$$
[\mathcal{E}, \mathcal{O}]=0 .
$$

Eq. (2) is a sufficient but not necessary condition of the exact transformation. If this condition is satisfied, the Hamilton operator in the FW representation is exactly defined by [2]

$$
\mathcal{H}_{F W}=\beta \epsilon+\mathcal{E}, \quad \epsilon=\sqrt{m^{2}+\mathcal{O}^{2}} .
$$

In this case, the transformation operator $U$ is described by

$$
U^{ \pm}=\frac{\epsilon+m \pm \beta \mathcal{O}}{\sqrt{2 \epsilon(\epsilon+m)}}
$$

where $U^{+} \equiv U, U^{-} \equiv U^{-1}$.

It follows from formulae (2),(3) that the operators $\beta \mathcal{O}^{2}$ and $\mathcal{E}$ commute with the Hamiltonian $\mathcal{H}_{F W}$. Therefore, wave eigenfunctions in the $\mathrm{FW}$ representation are also eigenfunctions

of the operators $\beta \mathcal{O}^{2}$ and $\mathcal{E}$. This circumstance simplifies considerably the determination of wave eigenfunctions.

\section{THE FOLDY-WOUTHUYSEN TRANSFORMATION OF SEVERAL OPER- ATORS}

Due to form (4) of operator $U$, some operators remain unchanged after the FW transformation for a stationary external field:

$$
\mathcal{E}_{F W}=\mathcal{E}, \quad\left(i \frac{\partial}{\partial t}\right)_{F W}=i \frac{\partial}{\partial t}, \quad \epsilon_{F W}=\epsilon .
$$

Eqs. (1)-(3) show that in the Dirac representation the operators $\mathcal{E}$ and $\epsilon$ commute with the Hamiltonian. Therefore, they have the eigenvalues, $\mathcal{E}_{0}$ and $\epsilon_{0}$ :

$$
\mathcal{E} \Psi=\mathcal{E}_{0} \Psi, \quad \epsilon \Psi=\epsilon_{0} \Psi
$$


The operators $\mathcal{E}$ and $\epsilon$ also commute with the Hamiltonian in the FW representation, $\mathcal{H}_{F W}$. The eigenvalues of these operators are defined by

$$
\mathcal{E} \Psi_{F W}=\mathcal{E}_{0} \Psi_{F W}, \quad \epsilon \Psi_{F W}=\epsilon_{0} \Psi_{F W}
$$

Certainly, the eigenvalues in Eqs. (6) and (7) coincide. For the particle energy operator, similar equations take the form

$$
i \frac{\partial}{\partial t} \Psi=\mathcal{H} \Psi=E \Psi, \quad i \frac{\partial}{\partial t} \Psi_{F W}=\mathcal{H}_{F W} \Psi_{F W}=E \Psi_{F W}
$$

\section{CONNECTION BETWEEN WAVE FUNCTIONS}

Let us analyze a connection between Dirac and FW wave functions when the FW transformation is exact. Eq. (1) leads to the formula

$$
i \beta \frac{\partial}{\partial t} \Psi=(m+\beta \mathcal{E}+\beta \mathcal{O}) \Psi
$$

Therefore, the connection between initial and final wave functions takes the form

$$
\Psi_{F W}=U \Psi=\frac{\epsilon+i \beta \frac{\partial}{\partial t}-\beta \mathcal{E}}{\sqrt{2 \epsilon(\epsilon+m)}} \Psi .
$$

Formulae (6),(8),(9) lead to the relation

$$
\Psi_{F W}=\frac{\epsilon_{0}+\beta\left(E-\mathcal{E}_{0}\right)}{\sqrt{2 \epsilon_{0}\left(\epsilon_{0}+m\right)}} \Psi
$$

This relation shows wave functions in the Dirac $\Psi=\left(\begin{array}{l}\phi \\ \chi\end{array}\right)$ and FW representations differ only by constant factors:

$$
\Psi_{F W}=\left(\begin{array}{c}
c_{1} \phi \\
c_{2} \chi
\end{array}\right)=\left(\begin{array}{cc}
c_{1} & 0 \\
0 & c_{2}
\end{array}\right)\left(\begin{array}{l}
\phi \\
\chi
\end{array}\right)
$$

Formulae (3),(8) result in

$$
(\beta \epsilon+\mathcal{E}) \Psi_{F W}=E \Psi_{F W} .
$$

In accordance with Eqs. (7),(11), either $E=\epsilon_{0}+\mathcal{E}_{0}$ or $E=-\epsilon_{0}+\mathcal{E}_{0}$. Since the total energy of particles is positive, first of these relations is right. As a result, Eq. (10) takes the form

$$
\Psi_{F W}=\frac{(1+\beta) \epsilon_{0}}{\sqrt{2 \epsilon_{0}\left(\epsilon_{0}+m\right)}} \Psi=\sqrt{\frac{2 \epsilon_{0}}{\epsilon_{0}+m}}\left(\begin{array}{l}
\phi \\
0
\end{array}\right)
$$


It follows from Eq. (12) that upper spinors in the Dirac and FW representations differ only by constant factors and lower spinors in the FW representation are equal to zero. Wave functions are normalized to unit. Therefore, we can renormalize the wave functions instead of calculation of the value $\epsilon_{0}$. As a result, the wave function in the FW representation can also be given by

$$
\Psi_{F W}=\frac{1}{\int \phi^{\dagger} \phi d V}\left(\begin{array}{l}
\phi \\
0
\end{array}\right) .
$$

In the considered case, a solution of wave equations in the FW representation is quite possible. Therefore, the connection between wave functions in two representations makes it possible to deduce FW wave eigenfunctions from Dirac wave eigenfunctions or on the contrary. In the Dirac representation, the lower spinor can be expressed in terms of the upper one.

\section{EXAMPLE: PARTICLE IN A UNIFORM MAGNETIC FIELD}

The FW transformation is exact for particles with an anomalous magnetic moment (AMM) moving in the plane orthogonal to a static uniform magnetic field [2]. In this case, the operator $p_{z}=-i(\partial / \partial z)$ commutes with the Hamilton operator and has eigenvalues $P_{z}=$ const. Therefore, the consideration of the particular case $P_{z}=0$ is quite reasonable [2].

The Hamilton operator in the Dirac representation satisfies Eq. (1) where

$$
\mathcal{E}=-\mu^{\prime} \Pi \cdot \boldsymbol{H}, \quad \mathcal{O}=\boldsymbol{\alpha} \cdot \boldsymbol{\pi}, \quad \boldsymbol{\pi}=\boldsymbol{p}-e \boldsymbol{A},
$$

$\mu^{\prime}$ is AMM, $\boldsymbol{A}$ is the vector potential, and $\boldsymbol{H}$ is the magnetic field strength.

In this case, the Hamilton operator in the FW representation is equal to [5]

$$
\mathcal{H}_{F W}=\beta \sqrt{\boldsymbol{\pi}^{2}+m^{2}-e \boldsymbol{\Sigma} \cdot \boldsymbol{H}}-\mu^{\prime} \boldsymbol{\Pi} \cdot \boldsymbol{H},
$$

where $\pi_{z} \Psi_{F W}=P_{z} \Psi_{F W}=0$ and

$$
\epsilon=\sqrt{\boldsymbol{\pi}^{2}+m^{2}-e \boldsymbol{\Sigma} \cdot \boldsymbol{H}}=\sqrt{\boldsymbol{\pi}_{\perp}^{2}+m^{2}-e \boldsymbol{\Sigma} \cdot \boldsymbol{H}} .
$$

The eigenvalues of the operators are defined by [6, 7]

$$
\begin{gathered}
\epsilon_{0}=\sqrt{m^{2}+(2 n+1)|e| H-\lambda e H}, \quad \mathcal{E}_{0}=-\lambda \mu^{\prime} H \\
n=0,1,2, \ldots, \quad \lambda= \pm 1 .
\end{gathered}
$$


Therefore, the connection between wave functions is given by Eq. (12), where $\phi$ is the upper spinor in the Dirac representation and $\epsilon_{0}$ is defined by Eq. (16).

Hamiltonian (15) commutes with the operators $\boldsymbol{\pi}_{\perp}^{2}$ and $\Pi_{z}$. Therefore, the wave eigenfunction $\Psi_{F W}$ is also an eigenfunction of these operators and can be given by

$$
\Psi_{F W}=\psi \zeta
$$

where $\psi$ is a coordinate wave function and $\zeta$ is an eigenfunction of operator $\Pi_{z}$ :

$$
\Pi_{z} \zeta=\lambda \zeta, \quad \lambda= \pm 1
$$

Since the lower spinors are zero, $\zeta^{+}=\left(\begin{array}{l}1 \\ 0 \\ 0 \\ 0\end{array}\right)$ when $\lambda=1$, and $\zeta^{-}=\left(\begin{array}{l}0 \\ 1 \\ 0 \\ 0\end{array}\right)$ when $\lambda=-1$.

We should take into account $z$-components of spin and orbital angular momentum have definite values. When $z$-component of total angular momentum equals $M$, the wave eigenfunctions take the form

$$
\begin{aligned}
\Psi_{F W}^{+} & =\frac{\exp [i(M-1 / 2) \varphi]}{\sqrt{2 \pi}} R_{|M-1 / 2|}(\rho) \zeta^{+}, \\
\Psi_{F W}^{-} & =\frac{\exp [i(M+1 / 2) \varphi]}{\sqrt{2 \pi}} R_{|M+1 / 2|}(\rho) \zeta^{-},
\end{aligned}
$$

where $R_{|M \pm 1 / 2|}(\rho)$ are the well-known radial eigenfunctions of operator $\boldsymbol{\pi}_{\perp}^{2}$ and the signs "+" and "-" mean positive and negative projections of spin, respectively.

For the particular case $P_{z}=0$, the wave eigenfunctions of Dirac particles with AMM in the uniform magnetic field derived in Ref. [7] can be obtained from Eq. (17).

\section{SUMMARY}

In this work, the connection between wave functions in the Dirac and Foldy-Wouthuysen representations has been found. When the FW transformation is exact, upper spinors in two representations differ only by constant factors, and lower spinors in the FW representation are zero. In the FW representation, relativistic formulae for operators are the simplest [1, 2]. Therefore, it is possible to use the wave eigenfunctions in the Dirac representation for calculating the mean values of operators in the FW representation. In other cases, the Dirac wave eigenfunctions can be deduced from the FW ones. 


\section{ACKNOWLEDGEMENTS}

I would like to thank Prof. V.A. Borisov for helpful discussion and to acknowledge a financial support by the BRFFR.

[1] L.L. Foldy, S.A. Wouthuysen, Phys. Rev. 78, 29 (1950).

[2] A.J. Silenko, J. Math. Phys. 44, 2952 (2003).

[3] D.M. Fradkin, R.H. Good, Rev. Mod. Phys. 33, 343 (1961).

[4] T. Goldman, Phys. Rev. D15, 1063 (1977).

[5] A.J. Silenko, Zs. Eksp. Teor. Fiz. 114, 448 (1998); [JETP 87, 246 (1998)].

[6] W. Tsai, Phys. Rev. D7, 1945 (1973).

[7] I.M. Ternov, V.G. Bagrov, and V.Ch. Zhukovsky, Vestn. Mosk. Univ. Fiz. Astron. No.1, 30 (1966); [Moscow Univ. Phys. Bull. No.1, 21 (1966).] 